\title{
LA CASA ESTA EN ORDEN. ANÁLISIS ESPACIAL INTRASITIO DE DELTA VULCANA 1 (LAGO MUSTERS, CHUBUT, ARGENTINA) Y MOVILIDAD DE CAZADORES-RECOLECTORES EN PATAGONIA CENTRAL DURANTE EL HOLOCENO TARDÍO
}

\author{
SANTIAGO PERALTA G.", J. EDUARDO MORENO“ \& HUGO PÉREZ R."
}

\begin{abstract}
RESUMEN
Este trabajo se propone conocer cómo se distribuye el registro arqueológico en el sitio Delta del Arroyo Vulcana 1 (lago Musters, Chubut) y de este modo, contribuir la comprensión de las pautas de movilidad y asentamiento que emplearon los cazadores-recolectores que habitaron en la región durante el Holoceno tardío.

Para tal fin se realizaron mapas de distribución y de densidad, mediante la utilización de un sistema de información geográfica. Los resultados obtenidos demostraron que existe una marcada estructuración intrasitio, y además permiten postular que en el sitio se llevaron a cabo tareas de ordenamiento y mantenimiento del espacio interno. Estos resultados aportan elementos a la discusión del modelo de alta movilidad residencial planteado para el Holoceno Tardío.
\end{abstract}

PALABRAS CLAVE: análisis espacial, intrasitio, cazadores-recolectores, Holoceno Tardío, lago Musters, Chubut.

THE HOUSE IS IN ORDER. INTRASITE SPATIAL ANALYSIS OF DELTA VULCANA I (LAKE MUSTERS, CHUBUT) AND MOBILITY OF GATHERERS-HUNTERS IN CENTRAL PATAGONIA DURING THE LATE HOLOCENE

\begin{abstract}
The main purpose of our article is to know how the archaeological register in Delta Arroyo Vulcana 1 (Musters Lake, Chubut) is distributed and with our conclusions help to understand the mobility and settlement of the hunter-gatherers that lived in the region during the Late Holocene.

For our work, we design distribution and density maps by using a geographic information system. The results shows that exists an intrasite structure, and support the idea that in that region prevailed resolutions that involved a model of order and maintenance of the internal space. These results provide elements within the discusion of high residential mobility in the Late Holocene.
\end{abstract}

KEY WORDS: spatial analysis, intra-site, hunter-gatherers, Late Holocene, Musters lake, Chubut.

FHyCS-UNPSJB y CENPAT-CONICET - CP 9005. peraltagonzalezsantiago@gmail.com; moreno@cenpat.edu.ar; hperezruiz@yahoo.com. 


\section{INTRODUCCIÓN}

El presente trabajo se inserta en el proyecto marco PI795-UNPSJB "Ocupaciones de cazadores-recolectores en la cuenca del Lago Musters: diversificación económica, movilidad y cronología”, dirigido por el doctor Julián Eduardo Moreno, a partir del cual se vienen realizando investigaciones desde el año 2005, y que busca conocer la antigüedad y características de la ocupación humana en Patagonia central. Entre sus objetivos se propone explorar indicadores de intensificación económica de grupos cazadoresrecolectores, producto de la explotación de recursos fluviales propios del lago (peces, coipos, aves, etc.) que den cuenta de posibles fenómenos de diversificación económica a lo largo del tiempo. Paralelamente, el proyecto marco evalúa la posibilidad de vincular la ocupación del lago Musters, con las de la costa, cordillera y la meseta de Patagonia central, a fin de examinar si esta cuenca jugó un papel significativo en el ciclo de movilidad de los grupos que la habitaron en el pasado.

En este trabajo se expondrán los resultados del análisis intrasitio efectuado en el sitio Delta Vulcana 1 (DV1), lago Musters, Chubut, Argentina. De todos los sitios relevados en la cuenca del lago Musters, DV1 es el que presentó la mayor potencialidad para el análisis estratigráfico.

\section{POBLEMÁTICA}

El análisis acerca del uso del espacio en tiempos pasados permite pensar cómo se configuraron las actividades humanas a través del tiempo (Binford 1995). En tal contexto, el estudio detallado del patrón de asentamiento como elemento analítico del registro arqueológico, hace posible el abordaje de una dimensión espacial de la complejidad y dinámica social. De esta forma, los distintos tipos de ocupación que pudieron producirse de manera recurrente o no en un mismo lugar, el modo de distribuir las actividades y de descartar los artefactos, y los procesos posdepositacionales, resultan en una configuración espacial particular del registro (Frank 2010; Mansur et al. 2007, Velázquez et al. 2007; Massonne et al. 1993).

Algunos autores consideran que los cazadores-recolectores de Patagonia meridional durante el Holoceno tardío se caracterizaban por una alta movilidad residencial (Miotti \& Salemme 2004). Paralelamente, otras investigaciones (Goñi \& Barrientos 2002) proponen, para los lagos del centro y Noroeste de Santa Cruz, una reducción de la movilidad residencial vinculada a un descenso de la humedad en la región, durante la Anomalía Climática Medieval (Stine 1994), ocurrida hace aproximadamente 1.000 años AP. Esta situación se habría traducido en un constreñimiento espacial de la población; y una ampliación del rango de acción, en términos logísticos y estacionales, hacia áreas altas circundantes (Goñi \& Barrientos 2002). En continuidad con esta última perspectiva, en la cuenca del lago Musters, el sitio DV1, de aproximadamente 1.400 años AP, aporta elementos a esta discusión.

De esta manera, como hipótesis general de trabajo se plantea que DV1 es un sitio arqueológico formado en condiciones de alta movilidad. Con el fin de dar cuenta de la gran concentración de materiales arqueológicos en el sitio, es posible desagregar la hipótesis propuesta en dos:

1. El sitio arqueológico DV1 es un palimpsesto en el sentido de Binford (1995), lo que implica una distribución aleatoria de los materiales, resultado de una sucesión de ocupaciones que no pueden ser diferenciadas entre sí.

2. DV1 se corresponde a la configuración de una ocupación breve, aunque intensa. De esta manera es esperable no encontrar una estructuración intrasitio, con áreas de actividad diferenciadas, como así tampoco evidencias de mantenimiento y acondicionamiento.

Descripción de la localidad delta del arroyo Vulcana y del sitio DV1

La localidad Delta del Arroyo Vulcana se sitúa en el margen oeste del lago Musters, al norte del cerro Pastel, y está atravesada por un fan-delta, originado por un arroyo proveniente de las sierras de San Bernardo. En la actualidad, el nivel del lago es más bajo que en el momento de la ocupación, por lo que el arroyo ha erosionado el sedimento deltaico dejando expuestos cortes que 


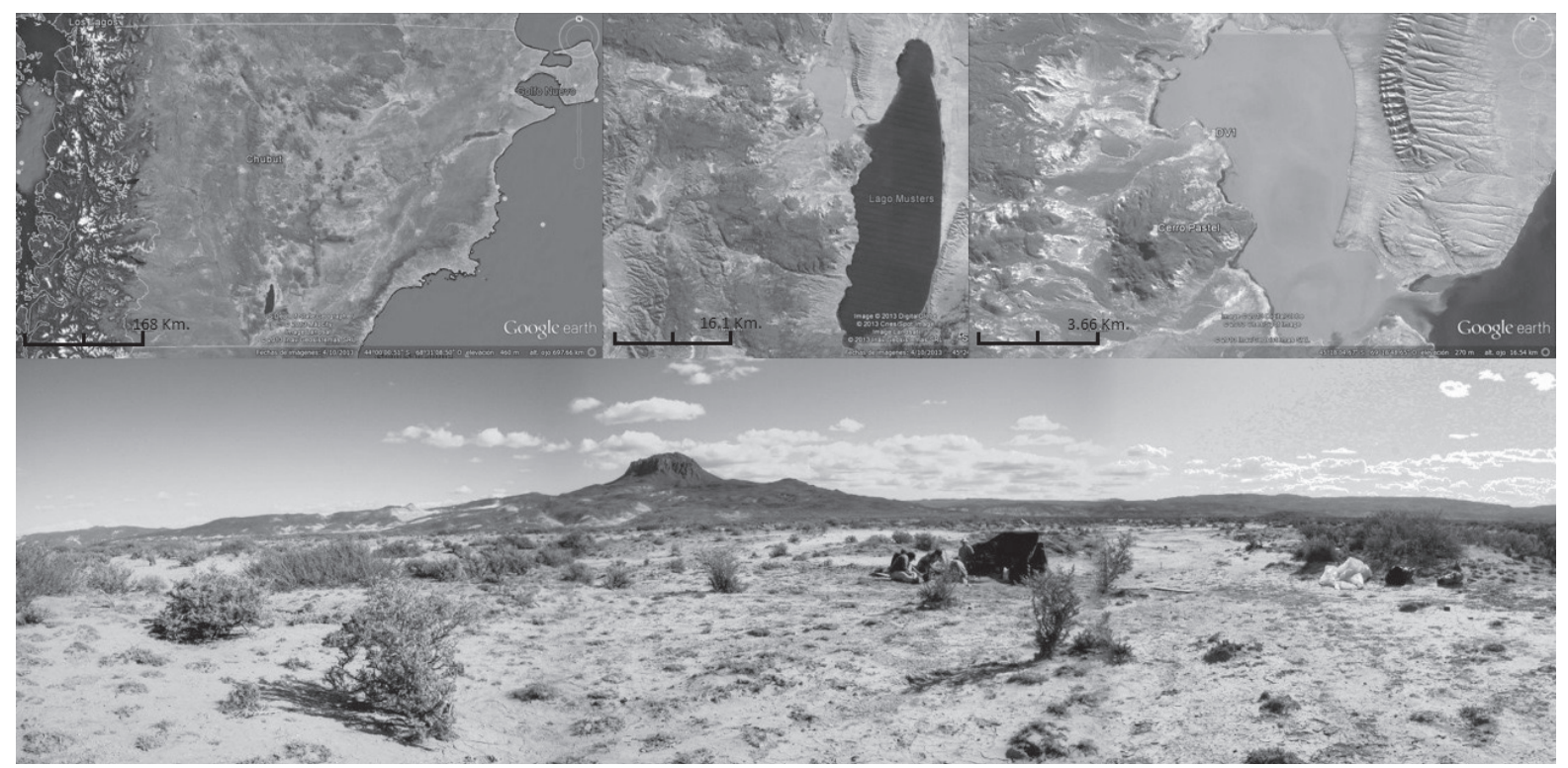

Fig. 1. Ubicación geográfica y visión general de sitio DV1.

superan el metro de profundidad por debajo del delta. Entonces, se trata específicamente de un paleo-fan-delta (Moreno et al. 2007, Moreno \& Pérez Ruíz 2010). Toda el área presenta evidencia de constantes fenómenos erosivos; reflejados en la presencia de vegetación en pedestal, en muchos casos con las raíces expuestas. La erosión permite una óptima visibilidad arqueológica en el terreno, y a la vez impacta negativamente sobre el registro. Si bien esta situación favorece la detección de sitios, requiere de una estrategia de trabajo que priorice los sitios en riesgo.

Las prospecciones realizadas pudieron detectar 32 sitios, definidos en el sentido de Borrero et al (1992), entre ellos DV1 (Moreno \& Pérez Ruíz 2010).

Dentro de la localidad se encuentra el sitio DV1, ubicado geográficamente en $45^{\circ} 17^{\prime} 23,7^{\prime}$ 'S69²0'05,8' O, comprendiendo el sector NE del frente del paleo fan delta, a 200 metros de la costa y a un metro sobre el nivel actual del lago

Se trata de un sitio subsuperficial a cielo abierto, cuya estratigrafía se compone de una capa superior arcillosa con lentes de arena y pequeños rodados, y una segunda capa arenosa con lentes de arcilla.

Durante el trabajo de campo se excavaron en 25 cuadriculas de $1 \mathrm{~m}^{2}$ cada una y un anexo de $1 \mathrm{~m}$ por $50 \mathrm{~cm}$, distribuidas en tres unidades:
- el sector norte: cuadriculas 1, 2, 3 y Anexo,

- el sector suroeste: cuadricula 4,

- el sureste: cuadriculas de la 5 a 26.

Las excavaciones realizadas detectaron importantes concentraciones de restos arqueofaunísticos, asociados a estructuras de combustión, artefactos líticos y probables huellas de poste.

La base de datos georreferenciada de DV1 presenta un total de 2319 elementos, correspondiendo 2157 (93,45\%) a material óseo y $152(6,55 \%)$ a material lítico, además de los cuatro fogones y probables huellas de poste.

La clasificación tipológica de los artefactos líticos señala como los artefactos más frecuentes a los raspadores $(n=17)$, filos naturales con rastros complementarios $(n=7)$, artefactos con retoque sumario $(n=4), y$, en menor cantidad, raederas y chuchillos. Se destaca la presencia de dos puntas lanceoladas, con hombros y de base cóncava. El conjunto lítico se completa con artefactos de piedra picada y/o pulida: tres fragmentos no diferenciados de molienda y una mano de molino trabajados sobre arenisca, un percutor, y un gran número de desechos de talla vinculados a las etapas finales del proceso de reducción (Reyes et al. 2012).

El análisis taxonómico del conjunto arqueofaunístico arrojó como especies principales al guanaco (Lama guanicoe) y a las percas 
(Percichtys sp). Los análisis realizados tanto en mamíferos como peces revelaron un bajo nivel de meteorización (Moreno \& Pérez Ruíz 2010; Svoboda 2011).

\section{Consideraciones teóricas}

La estructuración espacial de un sitio arqueológico refiere a la distribución particular que tienen los artefactos, las estructuras y los restos faunísticos en relación con la ocupación del mismo (Binford 1995). Esta estructuración intrasitio permite obtener información arqueológica que puede ser utilizada para elaborar hipótesis acerca de la función y organización de una ocupación. Según Binford (1995), tal configuración expone distintas áreas de actividad posibles de ser dotadas de significado como espacios donde se han realizado tareas específicas, o un conjunto de tareas relacionadas; estos clusters se caracterizan generalmente por una acumulación de productos de desechos y/o materia prima (Flannery \& Winter 1976). En el mismo sentido, y de acuerdo a la estratigrafía del sitio, también es factible asignarles a tales actividades una dimensión temporal.

Binford (1995) propone abordar el análisis de la distribución intrasitio de estos ítems de acuerdo a dos categorías de áreas de descarte: las zonas drop y toss. Las zonas drop son aquellos espacios de mayor actividad humana dentro del sitio $\mathrm{y}$, por los tanto, donde quedan depositados los restos de menor tamaño sin necesidad de ser trasladados a otra parte. Las zonas toss son aquellos sectores donde son arrojados los desechos de mayor tamaño, alejándolos de los espacios de mayor actividad humana. Del mismo modo, Schiffer (1972), postula una división entre descarte primario y secundario para referirse, por un lado, al material arqueológico que se desecha en el lugar en el cual fue producido $y$, por el otro, al que es concentrado en un lugar específico dando lugar a un área de descarte especializada.

Sobre la base de estas consideraciones y aplicando información georreferenciada se analiza la distribución del material arqueológico con software específico para el manejo de geodatabases.

\section{METODOLOGÍA}

Una de las ventajas de utilizar un Sistema de Información Geográfico (SIG) para el relevamiento de datos en arqueología reside en la capacidad de sistematización y manejo de grandes matrices de datos multidimensionales, esto es posible en razón del uso de técnicas complejas y algoritmos matemáticos, dando lugar a modelos o representaciones digitales del terreno (Buzai 2007).

De esta manera, los objetos individualizados en la excavación fueron organizados en una geodatabase de acuerdo a capas o niveles de información. El análisis de tal base de datos posibilitó construir un modelo vectorial del sitio dando cuenta de la posición espacial de cada artefacto relevado en 3 dimensiones. El proceso de sistematización se realizó para cada una de las cuadrículas individualmente, permitiendo al final contar con el modelo digital de cada una. En ese sentido fue posible observar una representación digital del contenido de todas las cuadrículas como si estuvieran abiertas al mismo tiempo.

Para la construcción del modelo digital del sitio DV1, y para la administración de datos se utilizó el software ArcGis 10 (Licencia acadénica FHyCS-UNPSJB). Como eje referencial se tomó con GPS un punto geográfico sobre un médano próximo al sitio, denominado Punto 0. Desde allí, con el uso de un nivel óptico, se trazaron las distancias hacia las cuadriculas excavadas, y sus respectivos rumbos. Esto permitió la construcción del modelo digital de las cuadriculas, a la vez que constituyó el marco para la georreferenciación de las fotografías digitales correspondientes a los distintos niveles de excavación.

Es posible interpretar a cada fotografía digital como una matriz de $\mathrm{n}$ dimensiones. De este modo, se obtuvo un mosaico de matrices de rasters que permitió conformar una base de datos georreferenciada a la cual fue posible agregarle campos adicionando nuevas dimensiones al análisis del sitio. Esta información raster constituyó el sustrato para la elaboración de una imagen vectorial de la planta, siguiendo los distintos niveles excavados y permitió representar el material arqueológico identificado con una serie de polígonos georreferenciados.

La información digitalizada de todos los 
artefactos y estructuras fue almacenada como información vectorial en una base de datos general del sitio. Los campos de interés para el relevamiento fueron: $\mathrm{n}^{\circ}$ de cuadricula, nivel, tipo de material, datos métricos. Paralelamente se complementó la base de datos con información específica del material lítico y óseo.

La elaboración de mapas de distribución general del material arqueológico del sitio constituyó uno de los primeros pasos de sistematización de la gran cantidad de datos obtenidos por cada nivel de excavación. Asimismo, se construyeron mapas de densidad de materiales por nivel y un mapa de densidad general del sitio que hizo posible vincular la información de los distintos niveles y, de esta manera, poner en evidencia sectores de concentración y sectores con bajo porcentaje de material arqueológico.

El análisis individual de los mapas de densidad por nivel de excavación permitió trazar una correspondencia entre los niveles superiores $e$ inferiores. Asimismo, se generaron mapas de densidad tanto para el material óseo, como para el material lítico con el fin de particularizar las concentraciones de ambos conjuntos y analizar la correspondencia. Además, se elaboraron mapas de los datos métricos del material, confrontando esta información con la de los mapas anteriormente mencionados. El análisis final dio lugar al examen de zonas de concentración diferencial de material, permitiendo indagar acerca de la posibilidad de existencia de distintas áreas dentro del sitio, que podrían estar vinculadas al descarte de material, o a distintos usos del sitio (Binford 1995).

\section{RESULTADOS}

\section{Formación del sitio DV1}

El sitio posee cuatro fechados radiocarbónicos de los fogones de las cuadrículas $1,4,5$ y 7 que arrojaron las siguientes dataciones respectivamente: $1310 \pm 70 \mathrm{AP}$ (LP-2081); 1490 \pm 70 AP (LP-2072); $1470 \pm 70$ AP (LP-2050) y $1490 \pm 90$ AP (LP-2028). De este modo, se pudo verificar que los fechados de las cuadrículas 4,5 , y 7 corresponden a un mismo periodo, lo que permitiría pensar que se produjo un único asentamiento.
Si se considera la ubicación cronológica de DV1 en 1.400 años AP aproximadamente, a priori se supone que el sitio fue generado en momentos de alta movilidad residencial. De este modo, en primer lugar, se analizó la posibilidad de que DV1 constituya un palimpsesto. En efecto, si DV1 fuese un palimpsesto la expectativa sería encontrar en el registro arqueológico una combinación de materiales de diferentes ocupaciones, con una distribución aleatoria, carente de algún tipo de estructuración interna, considerando al hombre como el principal agente de formación del sitio (Moreno \& Pérez Ruíz 2010)

Para evaluar la posibilidad de que DV1 constituya un palimpsesto, se realizaron cuatro mapas de densidad (correspondiéndo uno a cada nivel de excavación) de la distribución general, según el método Kernel, sin diferenciar entre artefactos líticos o arqueofaunísticos. De acuerdo a lo expresado anteriormente, se esperaría que la distribución del registro arqueológico presente un patrón espacial aleatorio $y$, de este modo, que no existan sectores claramente definidos en los cuales se concentren los artefactos. Como segunda expectativa, es esperable que pueda verificarse algún hiato en la distribución vertical, fundamentalmente, por tratarse de un contexto de rápida sedimentación.

Como se puede constatar en los mapas de densidad, las concentraciones se ubican en los mismos sectores de los cuatro niveles desde el superior hasta el inferior, es decir, que existe una misma tendencia en la disposición espacial del registro. Paralelamente, pudo determinarse la inexistencia de hiatos y que la cantidad de material disminuye gradualmente desde el nivel superior hacia el inferior. Estos dos datos permiten descartar la posibilidad de que el sitio DV 1 represente un palimpsesto. Además, hay que considerar que todos los artefactos se encuentran contenidos en una profundidad que no supera los $20 \mathrm{~cm}$. Resulta significativo destacar que gran parte del material se concentra en los primeros $10 \mathrm{~cm}$. Los gráficos de línea que se presentan a continuación demuestran que en el nivel 1 es en donde existe mayor cantidad de material. El número de restos decrece considerablemente para el nivel 2 y se vuelve mínimo tanto como para el nivel 3 como el 4 , lo que podría explicarse a partir de una migración 
Delta Vulcana 1-Sector SE

Mapa de densidad - nivel I DVI

Referencias

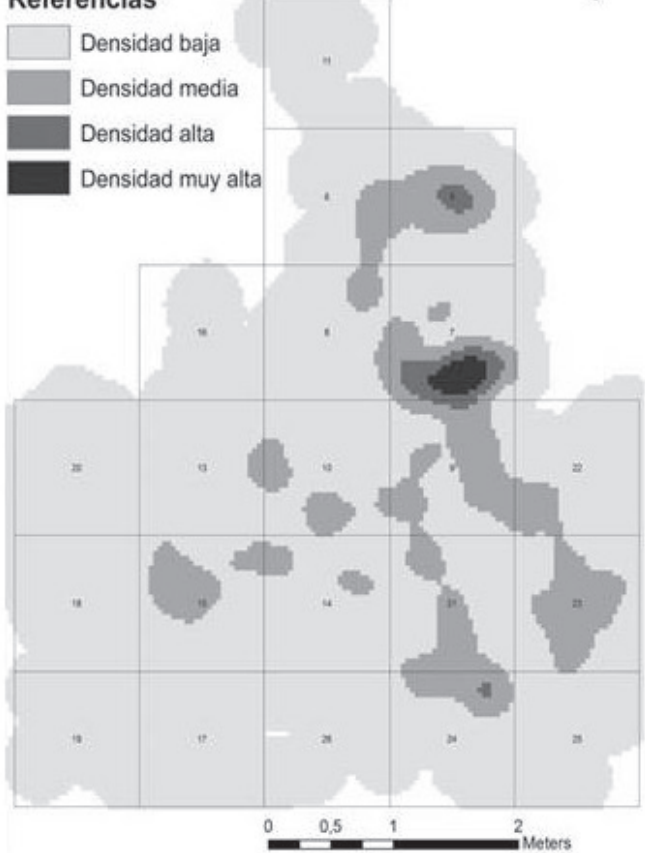

Delta Vulcana 1-Sector SE

Mapa de densidad - nivel 3 DVI

Referencias

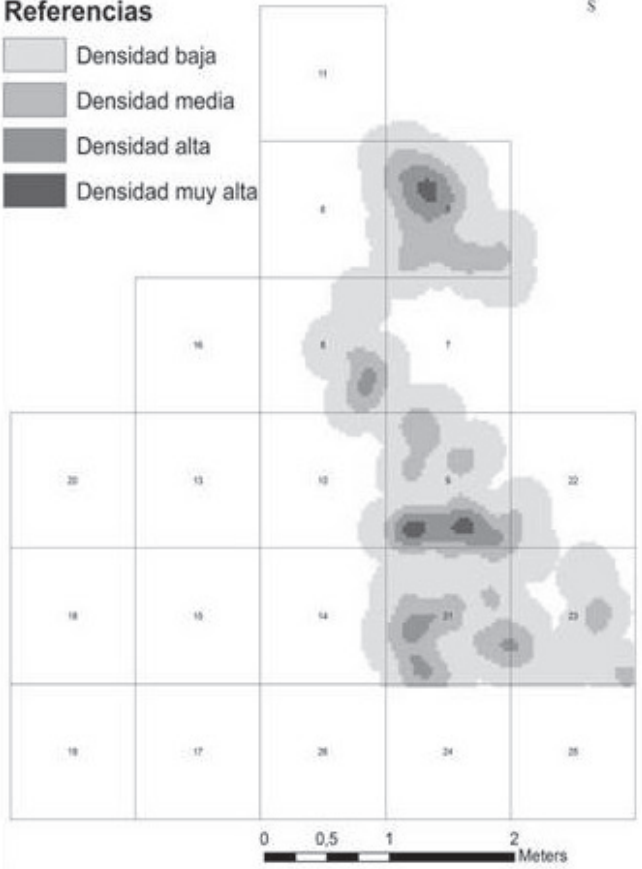

Delta Vulcana 1-Sector SE

Mapa de densidad - nivel 2 DVI

Referencias

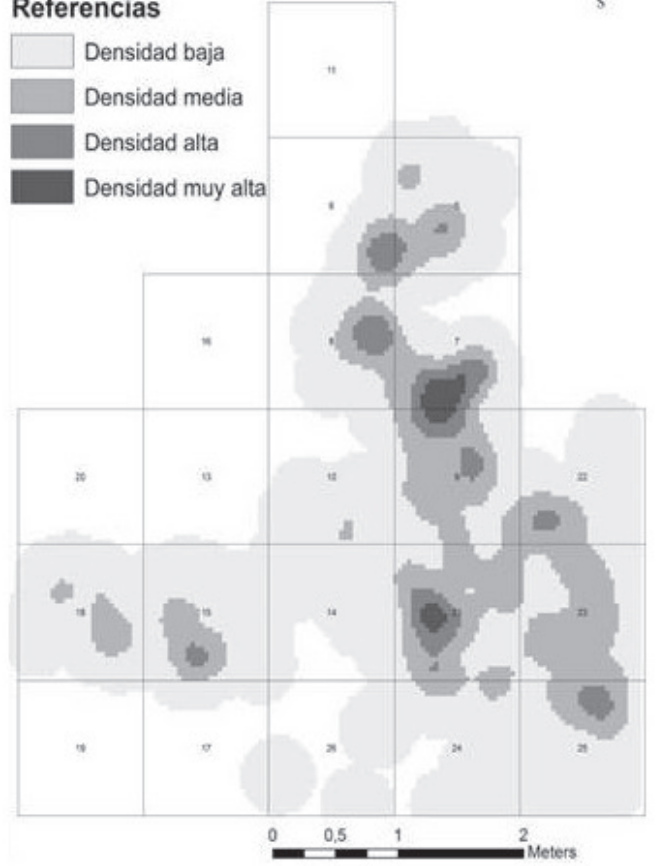

Delta Vulcana 1-Sector SE Mapa de densidad - nivel 4 DVI Referencias

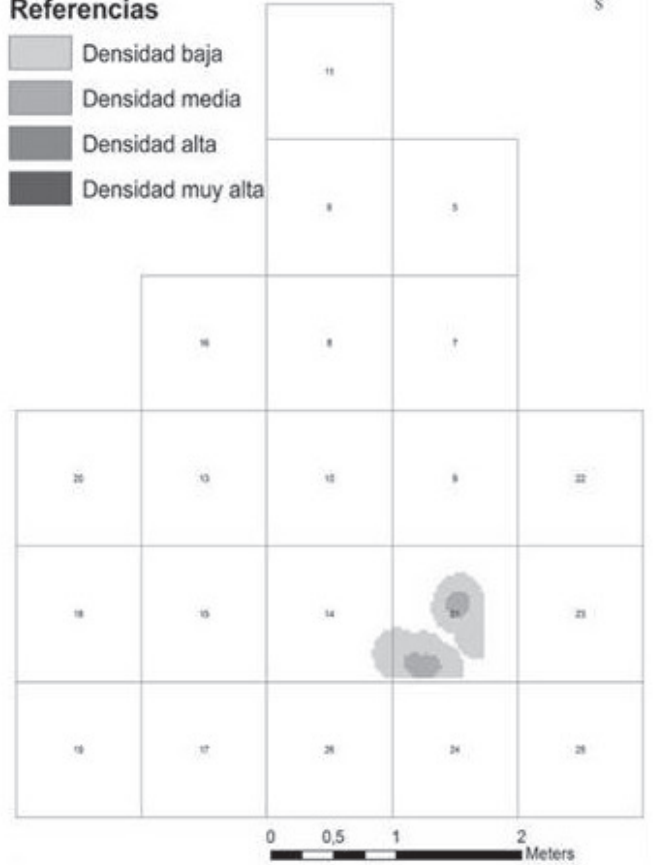

Fig. 2. Mapas de densidad de materiales arqueológicos por nivel de excavación. 
Tabla 1. Cantidad de materiales por nivel de excavación de acuerdo a su clasificación.

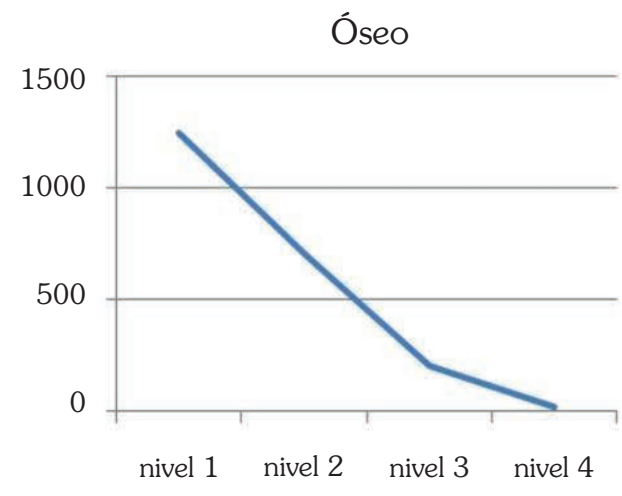

vertical.

Si bien la cantidad de restos óseos es mayor que la de la evidencia lítica, se observa en el gráfico de líneas que la tendencia de los dos conjunto es similar en cuanto a cómo disminuye el número de artefactos desde los niveles superiores a los inferiores.

El análisis tafonómico indica que en el conjunto arqueofaunístico se detectaron abundantes restos óseos de guanaco sin fusionar en posición articulada, como por ejemplo grupos de vértebras anatómicamente dispuestas con las carillas articulares no fusionadas. Esta información señala que el sitio no sufrió alteraciones significativas, es decir, no existió o fue escaso el movimiento posterior a la descomposición del tejido blando. Los materiales fueron cubiertos rápidamente probablemente en un periodo inferior a tres años posteriores a la ocupación, de acuerdo a los estudios experimentales realizados (Moreno \& Pérez Ruíz 2010, Svoboda 2013).

Lo desarrollado hasta aquí indica una única ocupación en el sitio DV1, o si ocurrió más de una, se produjo respetando el patrón de distribución horizontal previo, sin dejar evidencias en la distribución vertical, y sin alterar significativamente los restos óseos articulados. Cabe resaltar que se trata de un sitio a cielo abierto sin factores, al menos visibles, que permitan ordenar el espacio intrasitio, como podía ser en el caso de aleros o cuevas, por lo que resulta poco probable que esto haya ocurrido.

En cuanto al fechado del fogón de la cuadrícula 1 , si bien difiere respecto a las dataciones restantes, podría considerarse contemporáneo

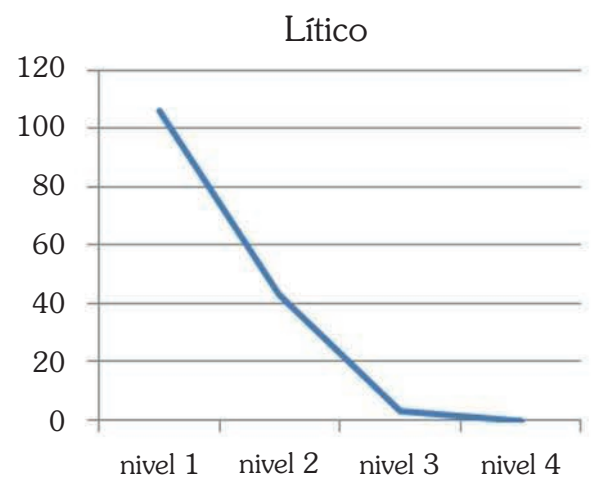

a las otras ocupaciones, ya que no implica una diferencia significativa. De todas formas, esta estructura de combustión se encuentra alejada de la concentración principal. Puntualmente, en esta investigación interesan las dos estructuras de combustión del sector Sureste de DV1 (cuadriculas 5 y 7$)$.

De acuerdo a lo desarrollado hasta el momento, existe una coherencia entre los resultados obtenidos de los análisis de distribución espacial, tafonomía, y cronología. Es decir, todo estaría indicando que en DV1 se habría llevado a cabo una ocupación única, o con menor probabilidad, una reocupación con una escasa separación temporal, y en ese caso, respetando el patrón espacial previo.

\section{Reconocimiento de rasgos arqueológicos generales}

Para detectar la existencia o no de estructuración espacial intrasitio, se tomaron los siguientes indicadores: huellas de poste, estructuras de combustión, y concentraciones de artefactos.

Las huellas de poste son el primer rasgo arqueológico que se reconoció, si se observa el sitio desde una perspectiva Noroeste-Sureste. Como se mencionó anteriormente, se detectaron cinco de este tipo de estructuras, formando una línea recta de $1,88 \mathrm{~m}$ con escasa distancia entre sí.

Para las huellas de poste se podría postular que su disposición se corresponde con la dirección predominante del viento, es decir en sentido Noroeste-Sudeste. Esta estructura respondería a la presencia de un paravientos, que permitía un refugio ante los vientos patagónicos. En las fuentes 
Tabla 2. Distancias entre huellas de poste.

\begin{tabular}{rccccc} 
& Huella 1 & Huella 2 & Huella 3 & Huella 4 & Huella 5 \\
\hline Huella 1 & - & $0,27 \mathrm{~m}$ & $1,07 \mathrm{~m}$ & $1,59 \mathrm{~m}$ & $1,87 \mathrm{~m}$ \\
\hline Huella 2 & $0,27 \mathrm{~m}$ & - & $0,78 \mathrm{~m}$ & $1,21 \mathrm{~m}$ & $1,51 \mathrm{~m}$ \\
\hline Huella 3 & $1,07 \mathrm{~m}$ & $0,78 \mathrm{~m}$ & - & $0,66 \mathrm{~m}$ & $0,76 \mathrm{~m}$ \\
\hline Huella 4 & $1,59 \mathrm{~m}$ & $1,21 \mathrm{~m}$ & $0,66 \mathrm{~m}$ & - & $0,28 \mathrm{~m}$ \\
\hline
\end{tabular}

etnográficas de Tierra del Fuego existen menciones a este tipo de estructuras habitacionales empleadas por los grupos Selk'nam (Gusinde 1982 [1931]).

El segundo indicador es la presencia de dos estructuras de combustión, ubicadas inmediatamente al Sudeste de las huellas de poste. El primer fogón se encuentra en las cuadriculas 5 y 6 , y posee una clara delimitación, es además de donde se recolectaron las muestras de carbón vegetal con mejor estado de conservación. El segundo fogón del sector Sureste, presenta una situación opuesta al primero. Esta estructura de combustión se encuentra $20 \mathrm{~cm}$ al Sur del primer fogón, entre las cuadriculas 7, 8, 9, 10, 13. 14, y 16 , y presenta poca claridad de su extensión lo que dificultó su delimitación.

Se ha postulado para las estructuras de combustión que el tamaño se vincula directamente con la intensidad de la ocupación. De este modo, cuanto más prolongado sea el asentamiento, más grandes y menos claros serán los límites del fogón, ya sea por las actividades de limpieza o por los procesos naturales que afectan el lugar (Chatters 1987; Massone et al. 1993). No obstante, hay que considerar que no siempre un grupo va a hacer uso de una estructura de combustión preexistente, lo que llevaría a la utilización de nuevos fogones, generando de este modo un conjunto de estructuras de combustión, que pudieron o no estar funcionando al mismo tiempo (Galanidou 2000).

Como se mencionó anteriormente, las estructuras de combustión de DV1 se encuentran asociadas a restos tanto óseos como líticos. Si bien, los materiales se encuentran en todo el sitio, las concentraciones su ubican al Este y Sur de los fogones.

A modo de resumen puede decirse que la distribución de rasgos arqueológicos detectados en el sitio presenta una disposición definida en sentido Noroeste al Sureste: las huellas de poste, le siguen las estructuras de combustión, y por último, las concentraciones de artefactos.

La disposición de las huellas de poste se encuentra oblicua a la dirección, Noroeste-Sureste,

\section{Delta del Arroyo Vulcana 1 (DV1)}

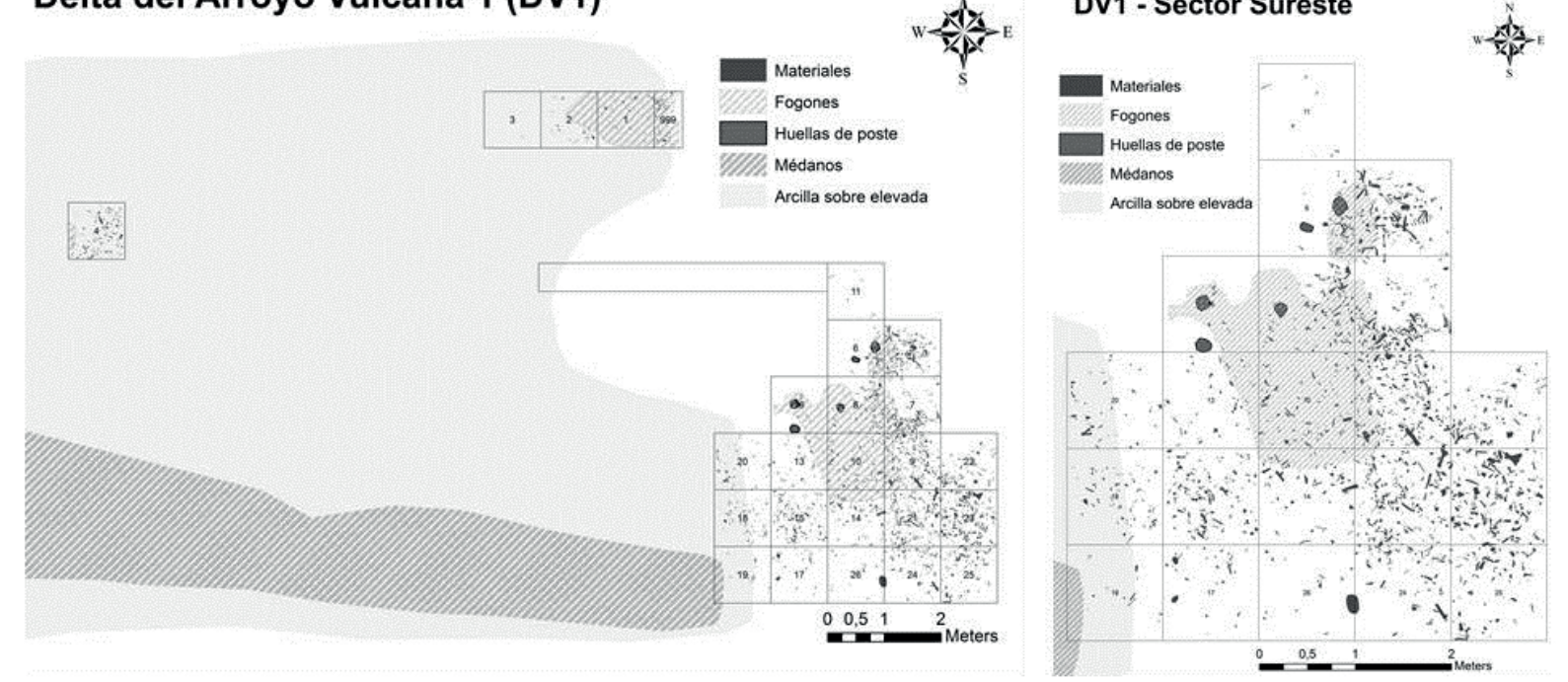

Fig. 3. Visión general del sitio y del sector sureste del sitio DV1. 

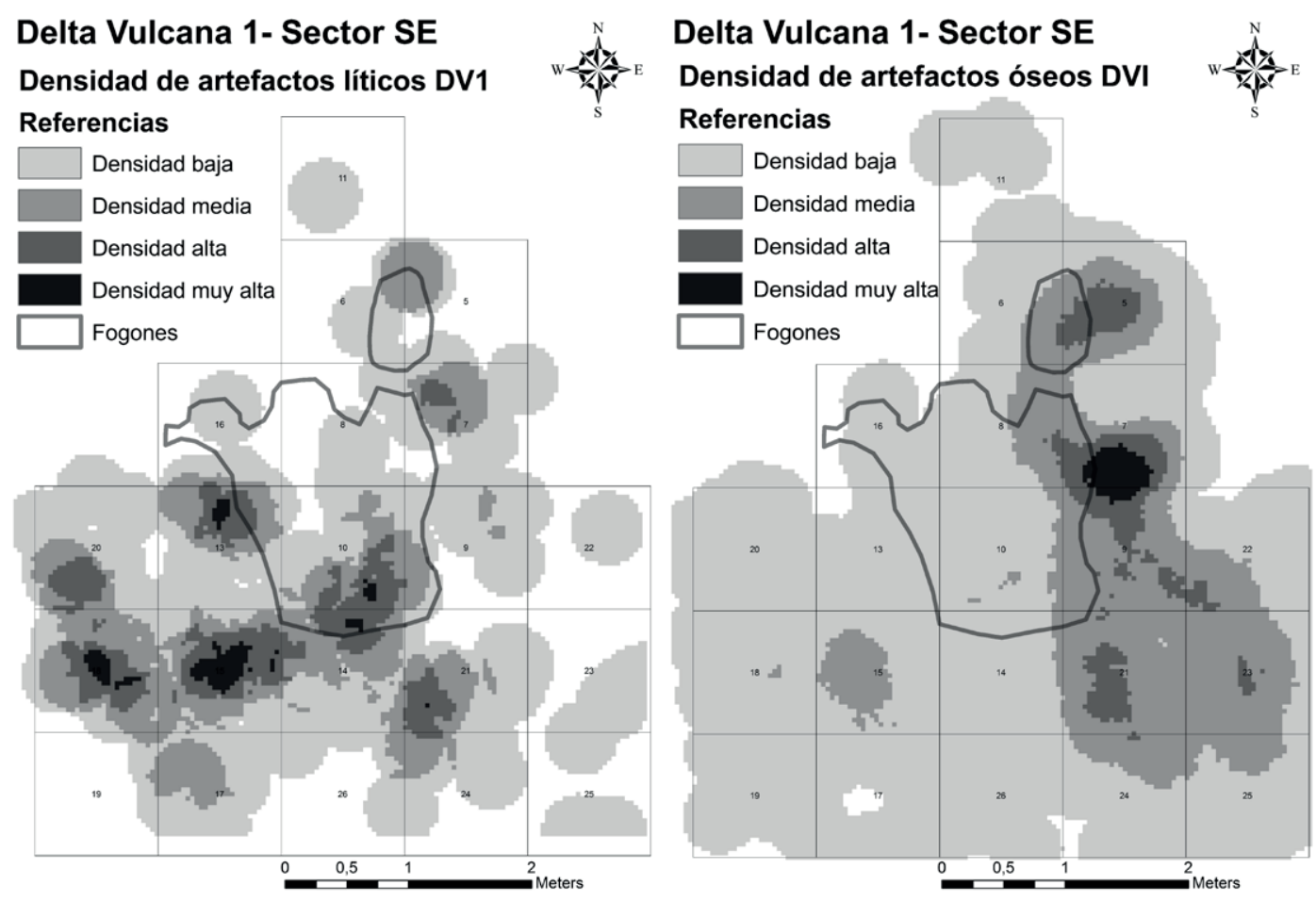

Fig. 4. Mapas de densidad del conjunto lítico y óseo.

predominante de los vientos. Por otro lado, las concentraciones de artefactos, tanto óseos como líticos, parecieran tener una pauta de distribución, centrada en su mayoría al Este y Sur de las estructuras de combustión.

\section{Distribución de artefactos líticos y óseos}

Para reconocer si existieron áreas de actividad (Flannery \& Winter 1976) en DV1, se realizaron análisis a partir de dos mapas de densidad, siendo el primero para artefactos óseos y el segundo para los líticos. En el primero se puede ver como rasgo principal que la tendencia general de distribución del conjunto arqueofaunístico tiende a concentrarse hacia el Este de las estructuras de combustión, sobre todo en las cuadriculas 5 y 7, y disminuyendo considerablemente al Oeste.

En el segundo mapa de densidad grafica del conjunto lítico del sitio, si bien existen pequeñas concentraciones en el margen Este, sin dudas el sector con mayor densidad de artefactos líticos del sitio es el Suroeste. Particularmente los mayores indices de concentración se dan en las cuadriculas $20,16,15,14$, y 10 , formando una figura que podría asimilarse a una " $U$ ", en donde se desarrollaron tareas de talla y descarte de artefactos líticos (Reyes et al. 2012).

Esto permite reconocer que los artefactos líticos poseen una distribución espacial diferente de los restos óseos, lo cual permitiría distinguir dos áreas de actividades.

Desde una perspectiva general, ninguna de las dos áreas de actividad se superponen, evidenciado así una posible planificación de las tareas que se desarrollaron en cada sector. Si se comparan las cuadriculas en donde se concentran los mayores índices de densidad por tipo de artefacto, 5 y 7 para restos óseos y 20,16, 15, 14, y 10 para lítico, podrá verse que están distanciados por no menos de 1 metro, y separados por las estructuras combustión, lo cual daría mayor sustento a la idea de una organización del espacio intrasitio.

$\mathrm{Si}$ se realiza una comparación entre los tres sectores excavadas que conforman DV1, es decir, el primero que incluye a las cuadriculas 1, 2, 3 y Anexo; el segundo formado por la cuadricula 4; y el último integrado por las cuadriculas que van de la 5 a la 26 , se comprueba que en general existe una distribución espacial similar en los tres sectores. En todos los casos 

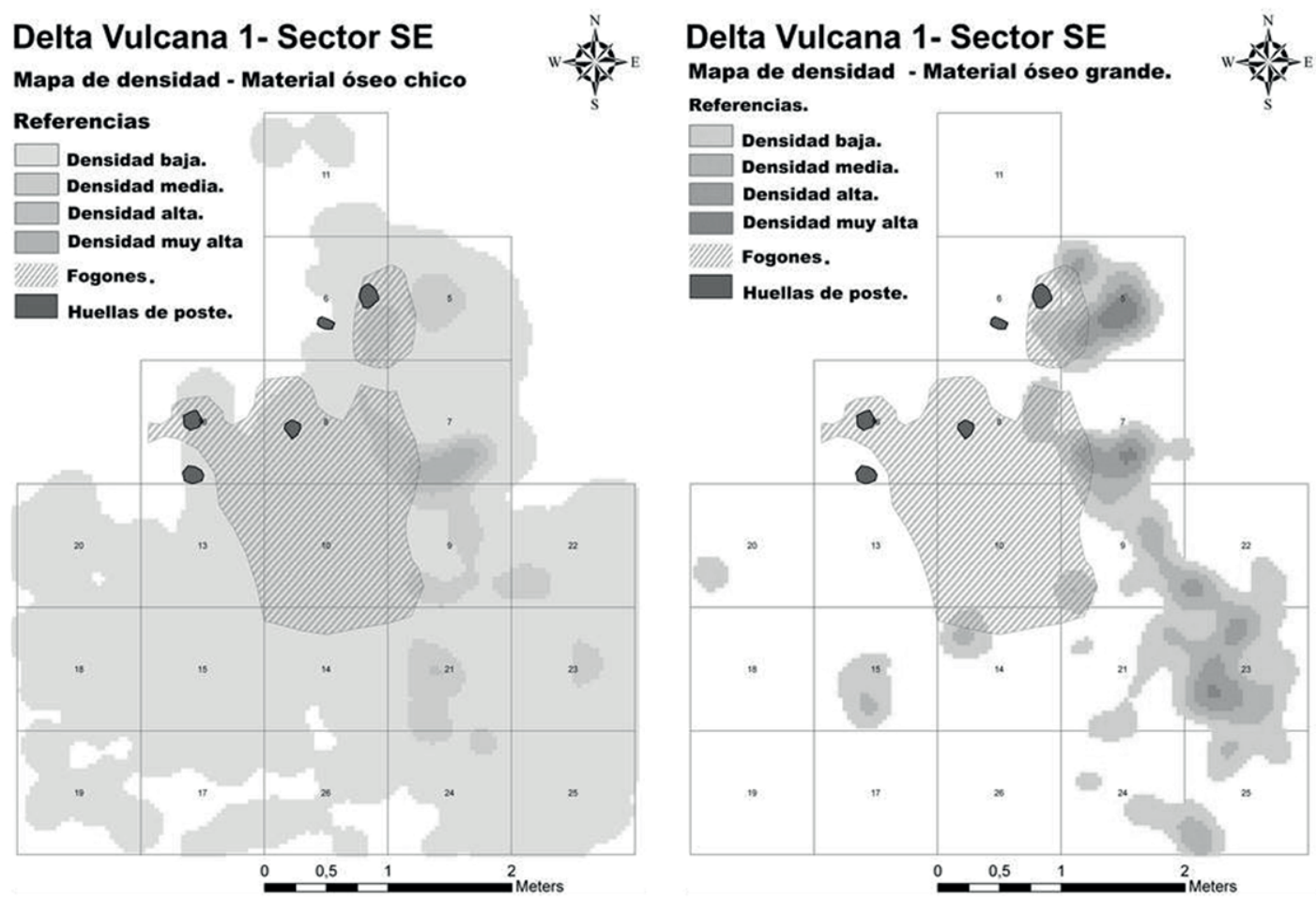

Fig. 5. Mapas de densidad del material óseo por tamaños.

se observa la existencia de estructuras de combustión y de restos óseos al Oeste de ellas, lo que estaría indicando una semejanza en cuanto a la disposición espacial del registro. Por otro lado, faltaría determinar si existen huellas de poste vinculadas a los fogones de los sectores Norte y Suroeste.

Resulta pertinente aclarar que tanto en el primero como el segundo sector a los cuales se hace referencia no han sido excavados en su totalidad, por lo que estas observaciones deben considerarse de manera parcial. De este modo, está previsto en futuras campañas realizar excavaciones en dichos lugares con el objeto de ampliar la información del sitio.

\section{Descarte y mantenimiento}

$\mathrm{Si}$ se retoma la hipótesis inicial, la expectativa de trabajo es no encontrar evidencias de acondicionamiento del espacio intrasitio en DV1, ya que cronológicamente se ubicaría en un momento de alta movilidad residencial, condiciones en las cuales no se efectuarían estas prácticas.

A priori, sería esperable que predomine un descarte del tipo primario por sobre uno secundario
(Shieffer 1972), es decir, detectar los artefactos en el lugar en el que fueron abandonados, evidenciando una escasa actividad de mantenimiento del espacio. Para ello se confeccionaron dos mapas en los cuales se establecieron sectores en los que se encontraron los artefactos óseos de menor y mayor tamaño. Esta clasificación se realizó solamente sobre el conjunto arqueofaunístico ya que la diversidad de tamaños de dichos restos facilitó detectar diferencias en la distribución espacial, condición que no se cumple en el conjunto lítico, volviéndose dificultosa la clasificación por tamaño.

Como se puede verificar en las figura 5, los ítems de menor tamaño se distribuyen de manera continua en todo el sitio, mientras que los de mayores proporciones se ubican en lugares puntuales, al Este de las estructuras de combustión. Esta disposición evidencia actividades de descarte diferencial en el espacio intrasitio, análogas a las zonas drop y toss (Binford 1995)

\section{DISCUSIÓN Y CONCLUSIONES}

Esta investigación se propuso aportar 
elementos a la discusión del modelo de movilidad y asentamiento para cazadores-recolectores durante el Holoceno tardío, en momentos previos a la Anomalía Climática Medieval, en la cuenca del Lago Musters. Si bien no existe información local sobre dicho fenómeno, se lo tomó como problemática de referencia.

De acuerdo a las hipótesis de trabajo presentadas, y a las expectativas que de ellas se desprenden, la investigación arrojó resultados que permiten arribar a las siguientes conclusiones:

En primera instancia, se planteó la hipótesis que sostenía que DV1 constituyera un palimpsesto. Esta expectativa de trabajo inicial fue descartada porque se pudo detectar que el registro arqueológico se concentra en los mismos sectores de los cuatro niveles de excavación, lo que permitió verificar una misma tendencia en la distribución espacial del material. Complementariamente, se determinó que cuantitativamente los artefactos disminuyen de modo pronunciado desde el componente superior hacia el inferior, fundamentalmente desde el nivel 1 al 2, volviéndose escasa la presencia de material en los niveles 3 y 4.

A los resultados arriba mencionados, habría que sumarles las conclusiones obtenidas en función de los estudios tafonómicos, de cobertura del sitio y cronológicos. Los primeros análisis postulan que DV1 no recibió alteraciones significativas posteriores a la ocupación. En cuanto a la cobertura del sitio se pudo establecer que DV1 fue rápidamente cubierto por procesos vinculados a una edafización o a una depositación de sedimentos eólicos (Moreno y Pérez Ruíz 2010).

La segunda hipótesis de trabajo planteada afirmaba que DV1 fue una ocupación breve. La información cronológica de las cuadriculas $1,4,5$ y 7 , indican que posiblemente se trata de una única ocupación, o en su defecto, se produjo una reocupación en un breve periodo, en donde se habría respetado la estructuración espacial del primer asentamiento. De este modo, la expectativa de trabajo era encontrar un sitio sin una estructuración interna, ni áreas de actividad diferenciadas, y carente de evidencias de mantenimiento del espacio intrasitio. En primera instancia, se pudo verificar la existencia de una estructura espacial intrasitio, conformada los siguientes indicadores: huellas de poste, estructuras de combustión, y concentraciones de artefactos, organizadas desde un eje Noroeste-Sureste.

Resulta importante observar que las huellas de poste están escasamente separadas de los fogones, de este modo no existe una distancia adecuada como para que el espacio comprendido entre estos rasgos arqueológicos haya sido un área habitacional. Si se realizara un buffer en función de lo propuesto por Nakazawa (2007), es decir, un modelo en el cual el radio calórico de una estructura de combustión posee $50 \mathrm{~cm}$ de alcance, se podría verificar que en DV1 es escasa la distancia entre las huellas de poste y los fogones. Si a esto se suma el hecho de que dos las huellas de poste se encuentran rellenas por cenizas, podría considerarse la posibilidad de que se haya cambiado la orientación de la estructura de refugio, de acuerdo a una variación en el rumbo de los vientos predominantes del sector, lo que constituye otro indicador de acondicionamiento del espacio intrasitio.

Por otro lado, ambos fogones se encuentran en asociación directa con restos arqueofaunísticos, cuya distribución espacial las ubica inmediatamente al Este de las estructuras de combustión, generando concentraciones similares en ambos fogones. Si bien las excavaciones aún no se han completado en los sectores Norte y Suroeste, los sondeos realizados parecerían indicar que los fogones y la evidencia arqueológica asociada presentarían la misma pauta de distribución espacial.

El tercer rasgo arqueológico lo constituyen las acumulaciones de artefactos, tanto restos de fauna como la evidencia lítica. En primera instancia pudo reconocerse que la mayoría del material arqueofaunístico en planta se ubica en el sector Este de DV1, en donde se encuentran los artefactos óseos de mayor tamaño. Si bien existen restos de fauna en todo el sitio, el sector que resulta significativo para reconocer la estructuración espacial intrasitio es el Este. Por otro lado, el conjunto lítico se dispone en su mayoría concentrado en el sector Suroeste del sitio, aunque también es pertinente observar que dicha evidencia se encuentra en todo DV1.

Fue posible detectar áreas de actividad diferenciadas, en las cuales se desarrollaron 
tareas específicas, como tallado de herramientas líticas y descarte de artefactos. La distribución diferencial de artefactos estaría indicando un acondicionamiento del espacio interno, dando lugar a sectores de descarte primario y secundario de artefactos. En el caso de DV1 puede postularse que los fogones constituyeron límites entre áreas de actividad y sectores de descarte primario y secundario. En este sentido, la organización del espacio intrasitio tomando como eje central a las estructuras de combustión fue desarrollada por Frank (2010) para la meseta central de Santa Cruz durante el límite entre el Pleistoceno final y el Holoceno temprano.

Sobre la base de la evidencia discutida hasta el momento, se postula que DV1 cumplió una función de campamento residencial, en el cual se pudieron detectar posibles áreas de actividades diferenciadas, una fuerte estructuración intrasitio, y evidencias de mantenimiento del espacio hacia el interior del asentamiento. Esto supone una tensión con respecto al modelo de alta movilidad, en momentos próximos a los 1.400 años AP. Esto no significa que el modelo de alta movilidad sea incorrecto, si no que al menos en el caso aquí analizado no cumplió con las expectativas esperadas. Quizás esto se deba a que DV1 sea un caso aislado, o que el modelo de movilidad que se empleó como problemática de referencia no sea aplicable a la cuenca del lago Musters.

Hasta el momento se han realizado sondeos en otros sitios del lago que no han arrojado resultados similares. De este modo, como perspectiva de trabajo se propone indagar si la estructura intrasitio que se plantea en el sitio DV1 es representativa del área o es un caso particular, y si tuvo algún tipo de implicancia a escala regional.

\section{AGRADECIMIENTOS}

A las instituciones que financiaron los trabajos de campo, UNPSJB y CENPAT-CONICET. A todos los miembros del equipo de Antropología y Arqueología de FHyCS-UNPSJB. A Rubén y Dolly por el apoyo logístico. Al Sr. Emir Abaid por permitirnos el ingreso a su estancia y al Sr. Nicolas Oviedo por su asesoramiento. A los evaluadores anónimos por sus valiosas sugerencias.

\section{BIBLIOGRAFÍA}

Binford, L. (1995). En búsqueda del pasado. Barcelona: Editorial Crítica.

Borrero, L. A., Lanata, J.L. \& Ventura, B.N. (1992). Distribuciones de hallazgos aislados en Piedra del Aguila. En L.A. Borrero \& J.L. Lanata (Eds.), Análisis espacial en la arqueología patagónica (pp. 9-20). Buenos Aires: Ediciones Ayllu.

Buzai, G.D. (2007). Dilemas de la relación Geografía-SIG entre la disciplina, interdisciplina, $y$ transdisciplina. GeoFocus, 7, 5-7.

Chatters, J. (1987). Hunter-Gatherer Adaptations and Assemblage Structure. Journal of Anthropological Archaeology, 6, 336-375.

Flannery, K. \& Winter, M. (1976). Research the household activities. En: K. Flannery (Ed.) The early Mesoamerican village (pp. 34-44). New York: Academic Press.

Frank, A. (2011). Tratamiento térmico y manejo del fuego en sociedades cazadoras-recolectoras de la Meseta Central de Santa Cruz. Doctorado, Universidad Nacional de La Plata, La Plata.

Galanidou, N. (2000). Patters in caves: forahers, horticulturists, and the use of space. Journal of Anthropoligical Archaeology 19: 243-275.

Gamble, C. (2002). Arqueología básica. Barcelona: Ariel.

Goñi, R. \& Barrientos, G. (2002). Poblamiento Tardío y movilidad en la cuenca del lago Salitroso. En: M.T. Civalero, P. M. Fernandez \& A. G. Guraieb (Eds.), Contra viento y marea. Arqueología de Patagonia ( pp. 313-324). Buenos Aires: Instituto Nacional de Antropología y pensamiento latinoamericano. Sociedad Argentina de Antropología.

Gusinde, M. (1982). Los indios de Tierra del Fuego. Tomo 1: los Selk'nam. Buenos Aires: Centro Argentino de Etnología Americana.

Mansur, M. E., Maximiano, A., Piqué, R., \& Vicente, O. (2007). Arqueología de rituales en sociedades cazadoras recolectoras. Una aproximación desde el análsis del espacio socialmente producido. En: F. Morello, M. Martinic, A. Prieto \& G. Bahamonde (Eds.), Arqueología de Fuego-Patagonia. Levantando piedras, desenterrando huesos... y develando arcanos (pp. 741-754). Punta Arenas: Ediciones CEQUA.

Massone, M., Jackson, D. \& Prieto, A. (1993). Perspectiva arqueológica de los Selḱnam. Santiago de Chile: Colección de Antropología I. Dirección de Bibliotecas, Archivos y Museos.

Miotti, L \& Salemme, M. (2004). Poblamiento, movilidad y 
territorios entre las sociedades cazadoras-recolectoras de Patagonia. Complutum, 15, 177-206.

Moreno, J., Videla, B., Pérez Ruíz, H., Asencio, L., \& Leonforti V. (2007). Búsqueda de Indicadores de Diversificación Económica Prehistórica en la Cuenca del Lago Musters, (Chubut, Argentina). Primeros Resultados. En: F. Morello, A. R. Prieto, M. Martinic \& G. Bahamonde (Eds.), Arqueología de Fuego-Patagonia. Levantando piedras, desenterrando huesos... y develando arcanos (pp. 22-32). Punta Arenas: Ediciones CEQUA.

Moreno, J. \& Pérez Ruíz H. (2010). Evidencias de utilización prehispánica de recursos fluviales en la cuenca del Lago Musters (Chubut, Argentina). En: J. Bárcena \& H. Chiavaza (Eds.) Actas del XVII congreso Nacional de Arqueología Argentina (pp. 345-350). Argentina: INCIHUSA-CONICET

Nakazawa, Y. (2007). Hearth-centered spatial organization: a comparative approach to the study of palimpsests in late upper Paleolithic sites in Hokkaido (Japan) and Cantabria (Spain). Doctorado, University of New Mexico, Albuquerque.

Reyes, M., Peralta González, S. \& López Ferrer A.L. (2013). Análisis preliminar de los materiales líticos del sitio Delta
Vulcana 1(Lago Musters, Chubut).En: A. F. Zangrando; R. Barberena; A. Gil; G. Neme; M. Giardina; L. Luna; C. Otaola; S. Paulides; L. Salgán \& A. Tivoli (Eds.), Tendencias teórico-metodológicas y casos de estudio de la arqueología en la Patagonia (pp. 219226). Buenos Aires: Museo de Historia Natural de San Rafael, la SAA y el INAPL.

Schiffer, M. (1972). Archaeological context and systemic context. American Antiquity 37, 156-165.

Stine, S. (1994). Extreme and persistent drought California and Patagonia during medieval time. Nature, 36, 546-549.

Svoboda, A. (2013). Disponibilidad cárnica, rendimiento energético y estimación de la talla de Percichthys trucha (perca criolla) a partir de la morfometría de huesos diagnósticos y su aplicación a los conjuntos ictioarqueológicos de Patagonia Central. Cuadernos de Antropología 9, En prensa.

Velázquez, H., Méndez, C., Reyes, O., Trejo, V., Sanhuesa, L., Quirós, D. \& Jackson, D. 2007. Campamentos residenciales tardíos a cielo abierto en el alto Río Cisnes (región de Aisen): Apeleg 1 (CIS 009). Magallania, 35, 121-132. 
